Ann. Biol. anim. Bioch. Biophys., 1979, 19 (1B), 251-256.

\title{
Transfert materno-fœetal et captation des acides gras essentiels chez le rat
}

par M. PASCAUD, H. PHAN, J. L. RENARD,

Physiologie méfabolique ef Nutrifion,

Université $P$. ef $M$. Curie, 75230 Paris Cedex 05.

Summary. Mother-fetus transfer and uptake of essential fatty acids by the rat fetus.

Transfer through the placenta and uptake of ${ }^{14} \mathrm{C}$ linoleic and arachidonic acids by the fetus were investigated in the 20 -day old rat fetus. ${ }^{14} \mathrm{C}$-labelled blood originating from pregnant rats fed ${ }^{14} \mathrm{C}$ methyl-linoleate was perfused in situ during $13 \mathrm{~min}$. into the placentofetal system.

A comparison of the plasma contents and of the ${ }^{14} \mathrm{C}$ specific activities of linoleic and arachidonic acids in different lipid classes in the mother and fetus plasma and the placenta led to the following conclusions : 1) the transfer of free fatty acids was fast and direct; 2) the triglycerides and cholesterol esters were lipolyzed when crossing the placenta; 3) the fetal plasma phospholipids were not an important vehicle in the transfer of arachidonic acid to the fetus as compared to triglycerides and cholesterol esters ; 4) fetal arachidonic acid originated essentially from the mother's liver in which it was actively synthesized.

Les acides gras polyénoïques de la famille linoléique sont indispensables au développement des animaux, notamment en tant que constituants structuraux des phospholipides des membranes cellulaires. En ce qui concerne les acides polyénoïques de la famille linolénique, ils apparaissent comme spécifiquement indispensables au développement des cellules nerveuses.

Alors que la circulation et la captation par le fotus de mammifère des acides gras non essentiels tels que l'acide palmitique ont fait l'objet de recherches nombreuses (Hummel et al., 1974, 1975 ; Elphick et Hull, 1977 ; Hudson et al., 1977) les recherches concernant les acides gras essentiels sont encore rares (Pascaud ef al.). Dans le présent travail, préliminaire à une investigation dynamique plus complète, on étudie le transfert materno-fœtal et la captation par le fœtus de raf âgé de 20 jours des acides essentiels linoléique $\left(18: 2^{w 6}\right)$ et arachidonique $\left(20: 4^{w 6}\right)$ apportés par le sang maternel après traversée du placenta.

\section{Conditions et méthodes expérimentales.}

L'investigation est réalisée in vivo par perfusion de courte durée in situ du système materno-fœtal d'une ratte Wistar âgée de 3 mois, gravide de 20 jours, nourrie à un 
régime standard équilibré (lipides 5 p. 100 dont acide linoléique 32 p. 100) par un sang maternel donné, apportant des acides gras marqués au ${ }^{14} \mathrm{C}$ de caractéristiques constantes.

Le sang marqué perfusant provient, par ponction aortique, de trois rattes également gravides de 20 jours ; il est prélevé $3 \mathrm{~h}$ après intubation stomacale forcée de linoléate de méthyle $1.14 \mathrm{C}(60 \mathrm{mCi} / \mathrm{mmole}$, Amersham). L'anticoagulant adopté est le citrate de $\mathrm{Na}$ à la dose $1,5 \mathrm{mg} / \mathrm{ml}$ (l'héparine entraîne l'hydrolyse rapide des triglycérides plasmatiques).

Le sang perfusant maternel est introduit dans le tiers inférieur du tronc aortique abdominal (pression $85 \mathrm{~mm}$, débit $2 \mathrm{ml} / \mathrm{mn}$ ) de la rate receveuse, non à jeûn, anesthésiée au nembutal. Le sang qui a irrigué la partie inférieure du corps de la ratte dont le système utéro-placento-fotal est recueilli par écoulement libre au niveau de la veine cave inférieure (fig. 1). L'expérience décrite rapporte les résultats d'une perfusion de durée $13 \mathrm{mn}$.

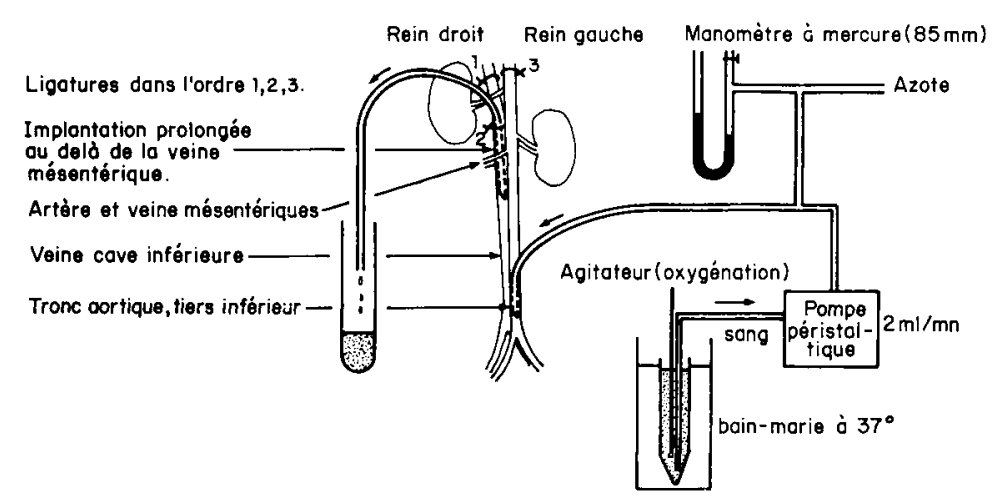

FIG. 1. - Perfusion in siłu du système placento-fœtal de la rate receveuse.

Les analyses de lipides, séparations chromatographiques sur colonne, sur plaque et en phase gazeuse, ont été décrites antérieurement (Pascaud ef al., 1977). La radioactivité ${ }^{14} \mathrm{C}$ est mesurée en scintillation liquide.

\section{Résultats et discussion.}

Après un examen comparatif des teneurs en acides polyénoïques linoléique et arachidonique des différentes classes lipidiques du plasma maternel perfusant et du plasma fœetal, on considérera, à l'issue de la perfusion, les niveaux d'activité spécifique ${ }^{14} \mathrm{C}$ de ces acides polyénoïques, indices de leur vitesse de transfert et de renouvellement.

1) Teneurs plasmatiques en acides linoléique et arachidonique. - D'après le tableau 1, il apparaît que les teneurs du plasma fœtal sont très inférieures aux teneurs maternelles. La différence est considérable pour l'acide linoléique des triglycérides respectivement 228 et $2422 \mathrm{nMoles} / \mathrm{ml}$ soit un facteur de 1 à 10 . Cette diminution résulte d'une rapide lipolyse dans le placenta (Edson ef al., 1975). 
2) Dynamique des transferts ef captations. - Le sang veineux recueilli à l'issue de la perfusion provient de la partie abdominale et postérieure de la rate receveuse et non pas du seul système placento-fœtal.

\section{TABLEAU 1}

Teneurs ( $\mathrm{nMoles} / \mathrm{ml}$ ) en acides linoléique ef arachidonique appartenant aux différentes classes de lipides plasmatiques de la mère ef du fœefus

\begin{tabular}{cccccccccc}
\hline \multirow{2}{*}{ nMoles $/ \mathrm{ml}$} & \multicolumn{2}{c}{ Acides gras libres } & \multicolumn{2}{c}{ Triglycérides } & \multicolumn{2}{c}{ Esters du cholestérol } & \multicolumn{2}{c}{ Phospholipides } \\
\cline { 2 - 9 } & Mère & Fœtus & Mère & Fœtus & Mère & Fœtus & Mère & Fœtus \\
\hline Acide linoléique ...... & 296 & 39 & 2422 & 228 & 224 & 65 & 34 & 35 \\
Acide arachidonique & .. & 41 & 26 & 202 & 57 & 385 & 83 & 146 & 34 \\
\hline
\end{tabular}

L'évolution en fonction du temps des teneurs du plasma veineux en acides libres linoléique et arachidonique (fig. 2) met en évidence deux phases successives : $a$ ) début de la perfusion : teneurs plus faibles correspondant au sang veineux préexistant dans la rate receveuse b) suite de la perfusion : augmentation due à l'apport du sang perfusant, les teneurs restant toujours inférieures d'un facteur de l'ordre de $1 / 2$ aux teneurs du sang perfusant.

L'évolution des activités spécifiques ${ }^{14} \mathrm{C}$ des acides linoléique et arachidonique dans le sang veineux recueilli (fig. 2) a la même allure dans les quatre classes lipidiques : après un bas niveau initial à l'exception de l'acide arachidonique libre, résultant d'une dilution importante par des acides froids d'origines plasmatique et tissulaire, augmentation rapide pour tous les lipides. Dans le cas des acides estérifiés cette augmentation tend à une équilibration avec le sang perfusant. Dans le cas des acides
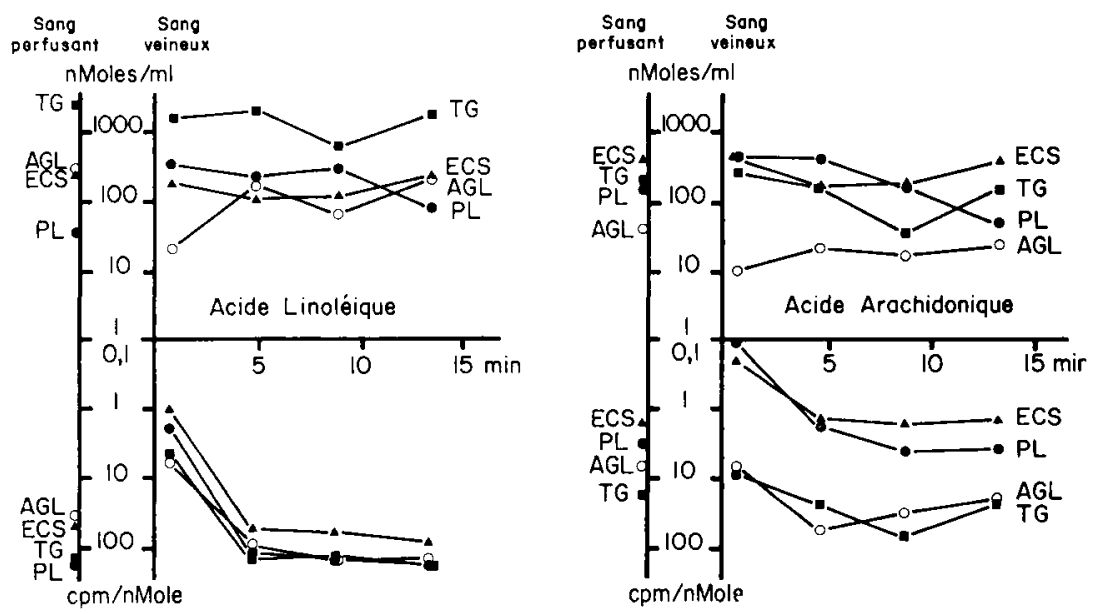

FIG. 2. - Evolution des teneurs et des activités spécifiques ${ }^{14} \mathrm{C}$ des acides linoléique ef arachidonique des différentes classes de lipides plasmatiques du sang veineux recueilli. AGL : acides gras libres; ECS : esters de cholestérol ; PL : phospholipides ; TG : tryglycérides. 
libres, les activités spécifiques sont supérieures aux activités respectives dans le sang perfusant, en raison d'une hydrolyse intra-plasmatique des triglycérides, à la fois plus concentrés et plus marqués.

La comparaison, à l'issue de la perfusion, des activités spécifiques des acides linoléique et arachidonique ainsi que palmitique, témoin non indispensable, dans les plasmas artériel maternel et total foetal (fig. 3) révèle également des comportements différents des acides gras libres d'une part et de tous les acides gras estérifiés d'autre part.

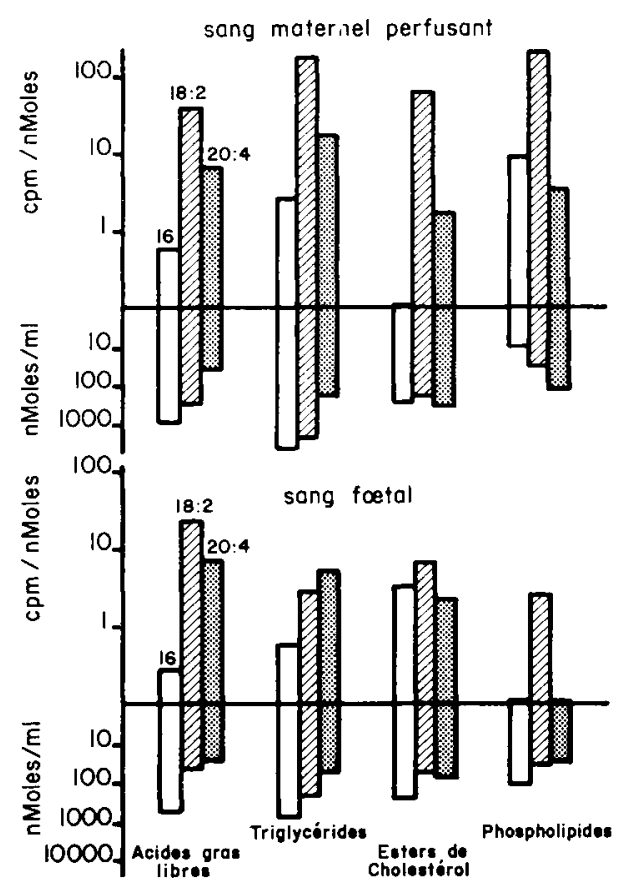

FIG. 3. - Teneurs plasmatiques et activités spécifiques ${ }^{14} \mathrm{C}$ des acides palmitique, linoléique et arachidonique des différentes classes lipidiques du plasma du sang maternel perfusant et du plasma "du sangifoétal après $13 \mathrm{~min}$. de perfusion.

L'ensemble des acides gras libres apparaît transféré directement avec toutefois une légère dilution des acides gras libres maternels par des acides gras libres placentaires et fœtaux moins marqués. En ce qui concerne les triglycérides la hiérarchie des activités spécifiques est inversée entre le plasma maternel et le plasma fœetal, l'acide linoléique étant fortement dilué par de l'acide linoléique moins marqué d'origine placenfaire et fotale. En ce qui concerne les phospholipides, l'atténuation considérable des activités spécifiques des trois acides résulte probablement du fait que le renouvellement des phospholipides plasmatiques du fœtus est plus lent que celui des autres classes lipidiques.

L'importance relative des différentes classes lipidiques pour le transport des acides gras dans le sang maternel ef dans le sang foetal est suggérée, à défaut de données concernant les vitesses de renouvellement, par la quantité de ${ }^{14} \mathrm{C}$ transportée 
dans chaque classe lipidique par $\mathrm{ml}$ de plasma. La juxtaposition des valeurs teneurs plasmatiques et activités spécifiques (fig. 3) représentées en coordonnées semi-logarithmiques, permet une représentation imagée.

Dans le sang maternel perfusant, les triglycérides dont le renouvellement est rapide apparaissent comme un transporteur actif des acides linoléique et arachidonique. Dans le sang fœtal, il s'agit à la fois des triglycérides et des acides gras libres, ces derniers provenant en partie de l'hydrolyse des triglycérides au cours de la traversée du placenta. Relativement aux autres classes lipidiques, les phospholipides du plasma foetal à renouvellement lent n'apparaissent pas comme un transporteur important de l'acide arachidonique.

3) Origine de l'acide arachidonique fœtal. - Le problème de l'origine maternelle ou foetale de l'acide arachidonique utilisé par le fœtus en développement peut être abordé par la comparaison des niveaux d'activités spécifiques d'une part de l'acide arachidonique, d'autre part de son précurseur acide linoléique, dans la carcasse du fœetus, dans le foie maternel connu comme synthétisant activement l'acide arachidonique et dans le foie fœtal (fig. 4).

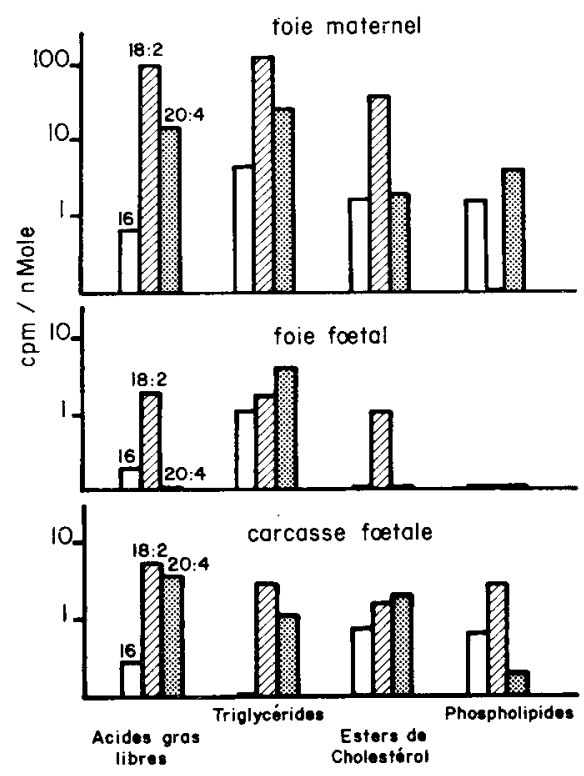

FIG. 4. - Activités spécifiques ${ }^{14} \mathrm{C}$ des acides palmitique, linoléique et arachidonique des différentes classes lipidiques du foie maternel, du foie foetal ef de la carcasse fœtale après $13 \mathrm{~min}$. de perfusion.

Alors que les niveaux d'activités spécifiques ${ }^{14} \mathrm{C}$ sont notables dans les phospholipides de la carcasse fœtale, bien que moins élevés que dans les autres classes lipidiques, ils sont infimes dans les phospholipides du foie fœtal. On remarque que l'acide arachidonique est également très peu marqué dans les acides gras libres du foie fœtal.

Dans le foie maternel au contraire, l'acide arachidonique est fortement marqué. Le fait que l'acide linoléique soit pratiquement froid dans les phospholipides du foie 
maternel est associé à une activité spécifique ${ }^{14} \mathrm{C}$ considérable (de l'ordre de $1000 \mathrm{cpm} /$ nMole) de l'acide gamma-linolénique dans la classe des phospholipides.

En ce qui concerne les triglycérides du foie fotal les niveaux ${ }^{14} \mathrm{C}$ élevés résultent de la captation et de la rétention des acides linoléique et arachidonique provenant des acides gras libres et des triglycérides plasmatiques.

L'acide arachidonique utilisé par le fœtus provient donc, au moins principalement, du foie maternel et non du foie fotal. Cette conclusion est en accord avec Strouve et Pascaud (non publié) et en désaccord avec Satomi et Matsuda (1973).

\section{Conclusion.}

L'ensemble des résultats acquis à l'issue de cette perfusion à court terme conduit aux conclusions suivantes : 1) le transfert de la forme acides gras libres est rapide et direci ; 2) les triglycérides subissent une hydrolyse importante au cours de la traversée du placenta ; 3) les phospholipides du plasma fœtal, à renouvellement lent, ne sont pas une forme significative de transport de l'acide arachidonique ; 4) l'acide arachidonique fœtal provient essentiellement d'une biosynthèse dans le foie maternel.

4e Réunion du groupe Développement I.N.R.A., Montpellier, 17-18 mai 1978.

Remerciements. - Ce travail a été réalisé avec la collaboration technique de J. Philippe et a bénéficié d'une subvention du Groupe Lipides ef Nutrition.

\section{Références}

EDSON J. L., HUDSON D. G., HULL D., 1975. Evidence for increased fatty acid transfer across the placenta during a maternal fast in rabbits. Biol. Neonate, 27, 50-55.

ELPHICK M. C., HULL D., 1977. Incorporation in vivo of $1{ }^{14} \mathrm{C}$ palmitic acid into placental and fetal liver lipids of the rabbit. Biol. Neonafe, 32, 24-32.

HUMMEL L., SHIRRMEISTER W., WAGNER H., 1975. Quantitative evaluation of the maternal-fetal transfer of the free fatty acids in the rat. Biol. Neonate, 26, 263-267.

HUMMEL L., SHIRRMEISTER W., ZIMMERMANN T., WAGNER H., 1974. Studies on the lipid metabolism using ${ }^{14} \mathrm{C}-1$-palmitate in fetal rats. Biol. Neonate, 24, 298-305.

HUDSON D. G., ELPHICK M. C., HULL D., 1977. Distribution of label in maternal plasma, placenta, fetal plasma and tissues after injection of ${ }^{14} \mathrm{C}$ palmitate into the circulation of 21 - and 28-day pregnant rabbits. Biol. Neonate, 32, 290-295.

PASCAUD M., ROUGIER A., DELHAYE N., 1977. Assimilation of ${ }^{14} \mathrm{C}$ linoleic acid by the rat fetus. Nutr. Metab., 21, 310-316.

SATOMI S., MATSUDA I., 1973. Microsomal desaturation of linoleic into linolenic acid in livers of fetal, suckling and pregnant rats. Biol. Neonate, 22, 1-8. 